\title{
DETECTION OF COLLISION EVENTS BY OLDER AND YOUNGER DRIVERS
}

\author{
George J. Andersen \\ Asad Saidpour \\ AnnJudel Enriquez \\ Department of Psychology \\ University of California, Riverside \\ Riverside, California, USA \\ E-mail: andersen@citrus.ucr.edu
}

\begin{abstract}
Summary: Recently (Andersen et al., 2000; 1998) we found that older drivers had poorer performance than younger drivers at detecting an impending collision during braking. In the present study we examined whether older drivers have poorer performance than younger drivers at detecting a collision with a moving object. 22 older and younger drivers were presented with computer generated scenes of a roadway in a driving simulator. Located in the scene was a single object that moved independently of the vehicle motion and that was or was not on a collision path with the vehicle. Overall older drivers were less sensitive to detect a collision than younger drivers, with performance worse for long as compared to short time to contact (TTC) conditions.
\end{abstract}

\section{INTRODUCTION}

An important perceptual task during driving is the ability to detect and avoid collisions. Failure to accurately detect collisions is a likely factor in accident risk. We have been examining the ability of drivers to detect collisions under a variety of conditions. In one study we assessed the ability of drivers to detect collisions during deceleration to a stop sign. Roadway scenes were computer generated and presented to the driver using a driving simulator. The scene simulated forward vehicle motion at a fixed speed toward a row of signs located in the center of the roadway. Following 6 sec of forward motion the vehicle decelerated at a fixed rate such that the vehicle stopped in front of the signs, at the signs, or beyond the signs (a collision). The computer scene was turned off prior to the vehicle reaching zero velocity and the driver's task was to determine whether or not the car would collide with the signs.

The results indicated that collision detection performance decreased with an increase in vehicle speed. In a follow up study (Andersen et al., 2000) we examined the detection capabilities of older drivers (mean age of 72). At slow speeds (30 mph) detection performance was only slightly worse for older drivers as compared to younger drivers. However, at higher speeds (60 $\mathrm{mph}$ ) collision detection was considerably worse for older drivers. The decrease in collision detection performance for older drivers was the result of an increase in the number of false alarms (reporting a collision to non-collision events) as well as a decrease in hits (reporting a collision to a collision event). In an additional study we found that the poorer performance of older drivers at the faster speed was the result of a decreased ability to use scene information to determine the speed of the vehicle.

In the present study we examined the ability of older and younger drivers to detect a collision with an oncoming moving object. The driving simulation displays depicted vehicle motion at a 
fixed speed and along a fixed trajectory. Within the scene a single object (a large ball) was moving at a fixed speed and along a fixed trajectory. On some trials the ball was on a collision path with the vehicle whereas on other trials the ball was not on a collision path. Similar to our previous research the display was turned off before the end of the event (a collision or the object passed outside the driver's field of view) and the driver's task was to determine whether the object was on a collision path.

Under these conditions a collision event is defined by two sources of information. One source of information is that the approaching object will expand in the visual field of the driver. This information is due to the decreasing distance between the object and vehicle and can be used to determine the time to contact of the object. The second source of information is that objects on collision paths with the vehicle will maintain a fixed location in the driver's field of view. Noncollision objects will translate to the left or right outside the field of view. This information is due to the constant relationship between the forward trajectory of the vehicle and the projected lateral location of the collision object.

It is important to note that the detection of a collision event during object and vehicle motion requires that both sources of information be present. Objects can be expanding in the field of view (e.g., objects that are passing to the left or right of the driver) of have a constant angular position (objects travelling in the same direction and at the same speed as the vehicle) and not be collision objects.

\section{METHOD}

Observers. The observers were 22 younger subjects (10 women and 12 men) from the University of California, Riverside campus and 22 older subjects (11 women and 11 men) from the Life Society Program at the University. All were paid for their participation and were naïve with regard to the purpose of the experiment. All observers were screened using several visual, perceptual and cognitive tests.

Design. Three within-subject independent variables were investigated: the TTC (2, 3.5, and 5 s), speed (24, 35, and $61 \mathrm{mph}$ ), and type of collision event (collision event or non-collision event). Age was run as a between subjects variable.

Stimuli. The displays simulated a three-dimensional scene consisting of a roadway, roadway strip, and a textured ground. The total dimension of the simulated space was 2000 by 1000 units. The roadway was 4 units horizontally and extended the entire length of the simulated space. A solid double-yellow line was projected down the length of the roadway. An irregular green texture pattern extended in all directions surrounding the roadway. The projection point of the scene was 1.2 units above the ground plane (This value represents 1 eyeheight in the scene). Within the scene were objects (bright red spheres of 0.5 unit radius) that translated within the scene. Each object was shaded (using a Gouraud shading model) to enhance the spherical shape.

The initial starting position of the object was located along an arc of fixed radial distance (115.2 units) from the simulated viewpoint. Each object translated in the scene at a fixed rate $(0.4$ units/frame) while the simulated viewpoint of the observer translated at a fixed rate $(0.2$ units/frame) down the center of the roadway. The display was updated at 20 frames/s. The 
trajectories of the translating objects were determined in the following manner. For noncollision objects an initial random trajectory was assigned that resulted in motion towards the simulated viewpoint. Next, the final position of the object, given the speed and trajectory of the object, was derived in accordance for the longest duration trial. If the final position of the object projected outside the field of view, a new random trajectory was selected. This constraint was used to ensure that non-collision objects remained in the field of view during each trial. A second constraint for non-collision objects was that the final position must project outside a circular region surrounding the simulated viewpoint. We refer to this constraint as the region of exclusion, which was either an 8 unit radius circular region.

Collision objects (targets) were defined in a similar manner to non-collision objects. Collision objects were assigned an initial random position along the fixed radial-distance arc. A trajectory was derived for the collision object such that it would intersect the simulated viewpoint given the speed of the object and the speed of observer motion.

Apparatus. The displays were generated using a Silicon Graphics Onyx Reality Station 10000 and presented, in a dark room, on a 50.8cm (resolution 1280 X 1024) non-interlaced color monitor. The total viewing distance to the monitor was $27.94 \mathrm{~cm}$ resulting in a visual angle of 40 deg.

Procedure. Subjects were informed that they would be shown a series of displays consisting of a roadway and surrounding field. The displays would simulate driving down the roadway. In the distance would be a single object. During the trial the object would move and their task was to determine whether the object would collide with the driver.

Observers were next shown a series of displays of collision and non-collision scenes to indicate the two types of events. Observers were shown the complete collision event during the instructions such that the object collided with the viewpoint (filled the display).

After observers understood the two types of events they were presented with 8 practice trials, half of which simulated a collision, and were asked to indicate whether or not the display simulated a collision. The practice trials depicted the complete event (collision or no collision). If the observer perceived no collision then they responded by pressing the right mouse button. If the observer perceived a collision event then they responded by pressing the left mouse button. No feedback was used. Observers were required to correctly identify 7 of the 8 practice trials before proceeding to the experimental trials. This was done to ensure that the subjects understood the task and response. 


\section{RESULTS}

The average proportion of hits (collision response for trials that simulated a collision) and false alarms (collision response for trials that did not simulate a collision) was calculated for each subject in each condition and used to derive a d' statistic (Green and Swets, 1966). The d'values for each subject in each condition were analyzed in a 3 (TTC) by 3 (speed) by 2 (age group) ANOVA

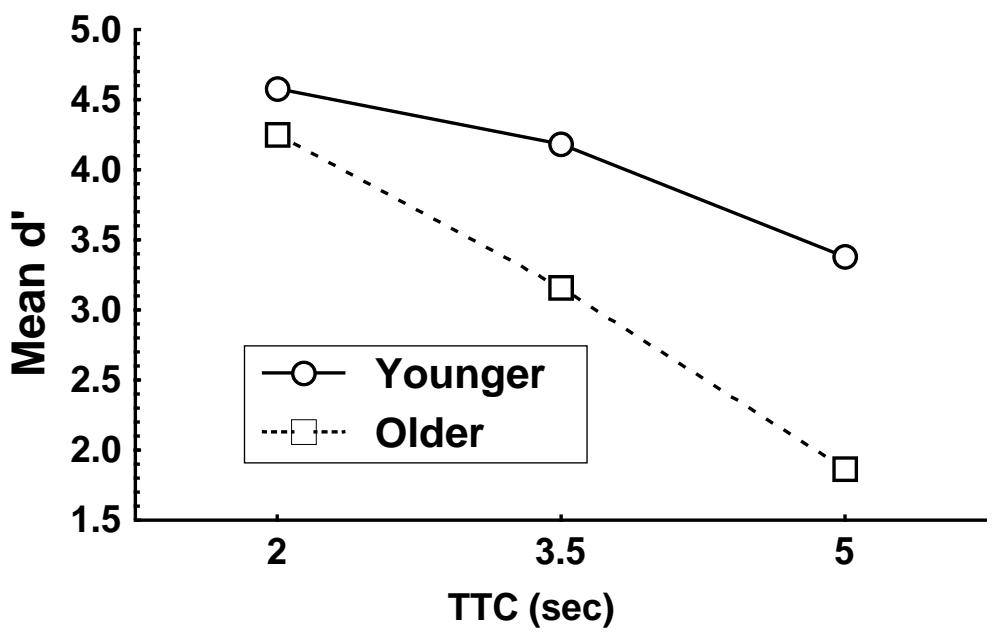
(Analysis of Variance). The main effect of age was significant, $\mathrm{F}(1,40)=11.74, \mathrm{p}<.01$, indicating that older observers were less

Figure 1. Collision detection performance as a function of age and time to contact (TTC).

sensitive (mean d' of 3.09) at detecting a collision than younger drivers (mean d' of 4.04). Age also interacted with TTC as shown in Figure 1.According to this result detection performance decreased with an increase in TTC, but the decrease in performance was much steeper for older drivers as compared to younger drivers. In order to increase TTC we increased the distance of the object from the vehicle in the simulation and maintained a constant speed. As a result, the size of the objects were much smaller for the $5 \mathrm{~s}$ TTC as compared to the $2 \mathrm{~s}$ TTC. This finding suggests that older drivers may have difficulty detecting a collision event for smaller objects in the driver's field of view.

\section{CONCLUSIONS}

In the present study we examined the ability of older drivers to determine whether a single object in a driving scene was on a collision path with the vehicle. The results indicate overall poorer performance for older than younger drivers. In addition, we found that older drivers had greater difficulty than younger drivers at detecting a collision for long TTC. This result may be due to a greater difficulty in detecting the motion of small objects in the driver's field of view.

\section{REFERENCES}

Andersen, G. J., Cisneros, J., Saidpour, A., \& Atchley, P. (2000). Age-related differences in collision detection during deceleration. Psychology \& Aging. 15, 241-252.

Andersen, G. J., Cisneros, J., Atchley, P., \& Saidpour, A. (1999). Speed, size, and edge-rate information for the detection of collision events. Journal of Experimental Psychology: Human Perception \& Performance. 25, 256-269. 